\title{
Open
}

\section{A mechanistic role for leptin in human dendritic cell migration: differences between ileum and colon in health and Crohn's disease}

\author{
HO Al-Hassi ${ }^{1}$, D Bernardo ${ }^{1}$, AU Murugananthan ${ }^{1}$, ER Mann ${ }^{1}$, NR English ${ }^{1}$, A Jones ${ }^{1}$, MA Kamm ${ }^{2,3}$, \\ N Arebi ${ }^{1}$, AL Hart ${ }^{1}$, AIF Blakemore ${ }^{4}$, AJ Stagg ${ }^{5}$ and SC Knight ${ }^{1}$
}

Dendritic cells (DC) migrate to lymph nodes on expression of C-C motif chemokine receptor 7 (CCR7) and control immune activity. Leptin, an immunomodulatory adipokine, functions via leptin receptors, signaling via the long isoform of receptor, LepRb. Leptin promotes DC maturation and increases CCR7 expression on blood DC. Increased mesenteric fat and leptin occur early in Crohn's disease (CD), suggesting leptin-mediated change in intestinal CCR7 expression on DC as a pro-inflammatory mechanism. We have demonstrated CCR7 expression and capacity to migrate to its ligand macrophage inflammatory protein $3 \beta$ in normal human ileal DC but not colonic or blood DC. In CD, functional CCR7 was expressed on DC from all sites. Only DC populations containing CCR7-expressing cells produced LepRb; in vitro exposure to leptin also increased expression of functional CCR7 in intestinal DC in a dose-dependent manner. In conclusion, leptin may regulate DC migration from gut, in homeostatic and inflammatory conditions, providing a link between mesenteric obesity and inflammation.

\section{INTRODUCTION}

Dendritic cells (DC) are heterogeneous, potent, antigenpresenting cells that comprise plasmacytoid and conventional/myeloid $(\mathrm{mDC})$ sub-populations. They are present as "immature" cells specialised for antigen uptake in most tissues of the body, particularly at the sites of interface with the external environment, such as the skin and mucosae. ${ }^{1}$ A property that distinguishes DC from other types of antigen-presenting cell is their potency in activating naive $\mathrm{T}$ cells, an interaction which generally occurs in secondary lymphoid tissue. Therefore, there is a requirement for antigen-bearing DC to migrate from the tissues to draining lymph nodes. In response to infection or signals that indicate local tissue damage, such as the production of pro-inflammatory cytokines, this migration of DC is accompanied by "maturation" in which antigen-acquisition machinery is downregulated, co-stimulatory molecule expression is enhanced and the ability to activate naive $\mathrm{T}$ cells is acquired. Furthermore, DC are present in the afferent lymph, draining the intestine even in the absence of overt inflammation, and a subset of these DC may be involved in nonresponsiveness. $^{2}$

Activation or "maturation" of DC populations generated in vitro is accompanied by a switch in the type of chemokine receptors expressed by the DC. ${ }^{3}$ Receptors for inflammatory-type chemokines, which are probably important for recruitment of immature DC into tissues in vivo, are downregulated upon maturation with lipopolysaccharide or tumor-necrosis factor $\alpha(\mathrm{TNF} \alpha)$. These receptors include C-C motif chemokine receptor 1 (CCR1), CCR2, CCR5, and CXCR1. By contrast, expression of CCR7, and migratory responses to its ligands, are strongly upregulated following maturation in vitro. ${ }^{3,4}$ CCR7 has three known ligands, CCL19 (macrophage inflammatory protein $3 \beta$ (MIP-3 $\beta)$ ) and two variants of CCL21 (6-C-kine), CCL21Leu and CCL21-Ser, expressed in lymph nodes or lymphatic endothelia. ${ }^{5}$

\footnotetext{
${ }^{1}$ Antigen Presentation Research Group, Department of Medicine, Imperial College London, Northwick Park and St Mark's hospitals, Harrow, UK. ${ }^{2}$ Department of Medicine, St Vincent's Hospital, University of Melbourne, Melbourne, Australia. ${ }^{3}$ Department of Gastroenterology, St Vincent's Hospital, University of Melbourne, Melbourne, Australia. ${ }^{4}$ Section of Investigative Medicine, Department of Medicine, Imperial College London, London, UK. ${ }^{5}$ Blizard Institute of Cell and Molecular Science, Barts and the London School of Medicine and Dentistry, Queen Mary University of London, London, UK. Correspondence: SC Knight (s.knight@imperial.ac.uk)
} 
Observations on DC matured in vitro have led to the chemokine receptor switch model, which proposes an activation-linked induction of CCR7, which is critical for the trafficking of DC to neighboring lymph nodes regardless of their maturation status. ${ }^{6}$ Whatever mechanisms drive the migration of DC in the absence of inflammation, these constitutively trafficking DC present tissue-specific self-antigens. ${ }^{7}$ They can have a crucial role in maintaining tolerance by deleting or silencing potentially autoreactive $\mathrm{T}$ cells that have escaped thymic selection. ${ }^{8}$

A characteristic symptom of Crohn's disease (CD) is hypertrophy of mesenteric fat and fat wrapping the intestine. This "creeping" fat is the secretion site of biologically active molecules, known as adipokines, ${ }^{9,10}$ which act on the immune system; these adipokines include leptin, adiponectin, resistin, and visfatin. Leptin is primarily produced by adipocytes and is a regulator of appetite, limiting food intake. However, leptin also has a role in regulating immune responses. ${ }^{11}$ For example, it promotes maturation and survival of DC by activating nuclear factor- $\kappa \mathrm{B}$, which has an anti-apoptotic effect on DC. ${ }^{12}$ Leptin also improves the ability of DC to stimulate $\mathrm{T}$ cells and promote a T helper type 1 type response. ${ }^{13,14}$ Furthermore, treatment of monocyte-derived DC with leptin increases production of interleukin (IL)-12, IL-6, and IL-1b and downregulates the production of IL-10, underlining the view of leptin as a proinflammatory adipokine. ${ }^{12}$ Hence, studies on leptin function outside the neuroendocrine axis by targeting cells of the immune system via its receptor in health and disease has been suggested. ${ }^{15}$ Leptin signals via leptin receptors (LepRs), which exist in several isoforms (LepRa-f); these receptors have identical extracellular domains, but only the long isoform LepRb has a long cytoplasm domain and definite signaling capacity through the Janus kinase (JAK)/STAT (signal transducer and activator of transcription factor) pathway ${ }^{16,17}$ and phosphorylation of STAT3 (pSTAT3). The other isoforms LepRa, $\mathrm{c}-\mathrm{f}$ have shorter cytoplasm domains and may signal through the mitogen-activated protein kinase pathway. ${ }^{18,19}$ The expression pattern of LepRb in the colon and terminal ileum, and the effects of leptin on DC migration in relation to $\mathrm{CD}$ are still largely unknown.

Accumulating evidence has linked the effect of leptin to DC migration. For example, leptin upregulates the expression of CCR7 on immature, peripheral blood DC. ${ }^{20}$ Moreover, mature DC from leptin receptor-deficient mice expressed low levels of CCR7, indicating an impaired migratory function. ${ }^{21}$ In fact, leptin and CCR7 signaling have several interlinked functions on DC; similar to leptin, signaling via PI3-Akt, ${ }^{22}$ CCR7 prolongs DC lifespan by activating the anti-apoptotic effect of nuclear factor- $\kappa \mathrm{B} .{ }^{23}$ They both induce morphological changes in DC by inducing regulation and rearrangement of actin microfilaments of the DC cytoskeleton leading to an increase in their veiled extensions, indicating a positive effect on DC migration and capacity to capture and stimulate $\mathrm{T}$ cells in the lymph nodes. $^{12,24}$ These findings suggest a special relationship between immune cells and mesenteric fat. They raise questions about local changes in leptin/leptin receptor production in inflammatory bowel disease (IBD), including whether leptin has an immunomodulatory effect on DC function in the gut.

The trafficking of DC populations that sample and respond to microbial products at different sites in the intestine and the regulation of this trafficking is likely to be a control point in intestinal responses. However, most studies of gut immunity are performed in the mouse and more particularly-when details are given-in the mouse ileum. Extrapolating findings by generalizations about "intestinal immunity" when only studying the microbe-poor environment of mouse ileum and not the microbe-rich colonic regions and in pathogen-free animals on a defined diet may not be accurate models for human immunity in different regions of the gut. Thus, we studied, in humans, the expression and function of CCR7, both in DC from the microbe-rich colonic environment and in DC from the relatively microbe-poor terminal ileum. We also examined whether the action of leptin via LepRb provided a mechanism for functional CCR7 induction. The possible involvement of this pathway in gut inflammation in IBD was also examined. The results provide evidence that stimulation of CCR7 expression by leptin is involved in homeostasis in the ileum but is operating in the colon exclusively in IBD. Hence, DC trafficking is differentially regulated in the colon and terminal ileum and alterations in trafficking may contribute to pathology in IBD.

\section{RESULTS \\ Expression of functional CCR7 by healthy blood and intestinal human DC}

Blood DC. Multi-color flow cytometry was used to identify DC and to examine CCR7 expression on DC in fresh whole blood. DC were identified as shown in the Methods section (Figure 1a), and CCR7 was not detectable on $\mathrm{mDC}$, whereas, blood plasmacytoid DC expressed CCR7 (Figure 1b).

Intestinal DC-expression of CCR7 differed between ileum and colon. CCR7 was not detected on most freshly isolated DC from colonic samples of non-inflamed tissue from control individuals, but expression was increased on DC from normal ileum $(P<0.0001$; Figure 2a). Furthermore, in paired samples from the same individuals, the expression of CCR7 was significantly higher on DC from normal ileum compared with that on normal colonic DC $(P<0.001$; Figure 2b). Functional studies showed that ileum DC that expressed CCR7 migrated towards MIP-3 $\beta$, a CCR7 receptor chemo-attractant, in chemotaxis assays and that this migration was concentration dependent (Figure 2c).

\section{Production of LepRb by ileal and colonic human DC}

To assess whether signaling through the leptin receptor might contribute to the CCR7 expression on DC, we determined the ongoing production of LepRb by measuring intracellular accumulation of the LepRb protein in a 4-h in vitro culture of freshly obtained DC. Production of LepRb mirrored the expression of CCR7. Thus, there was minimal or undetectable 

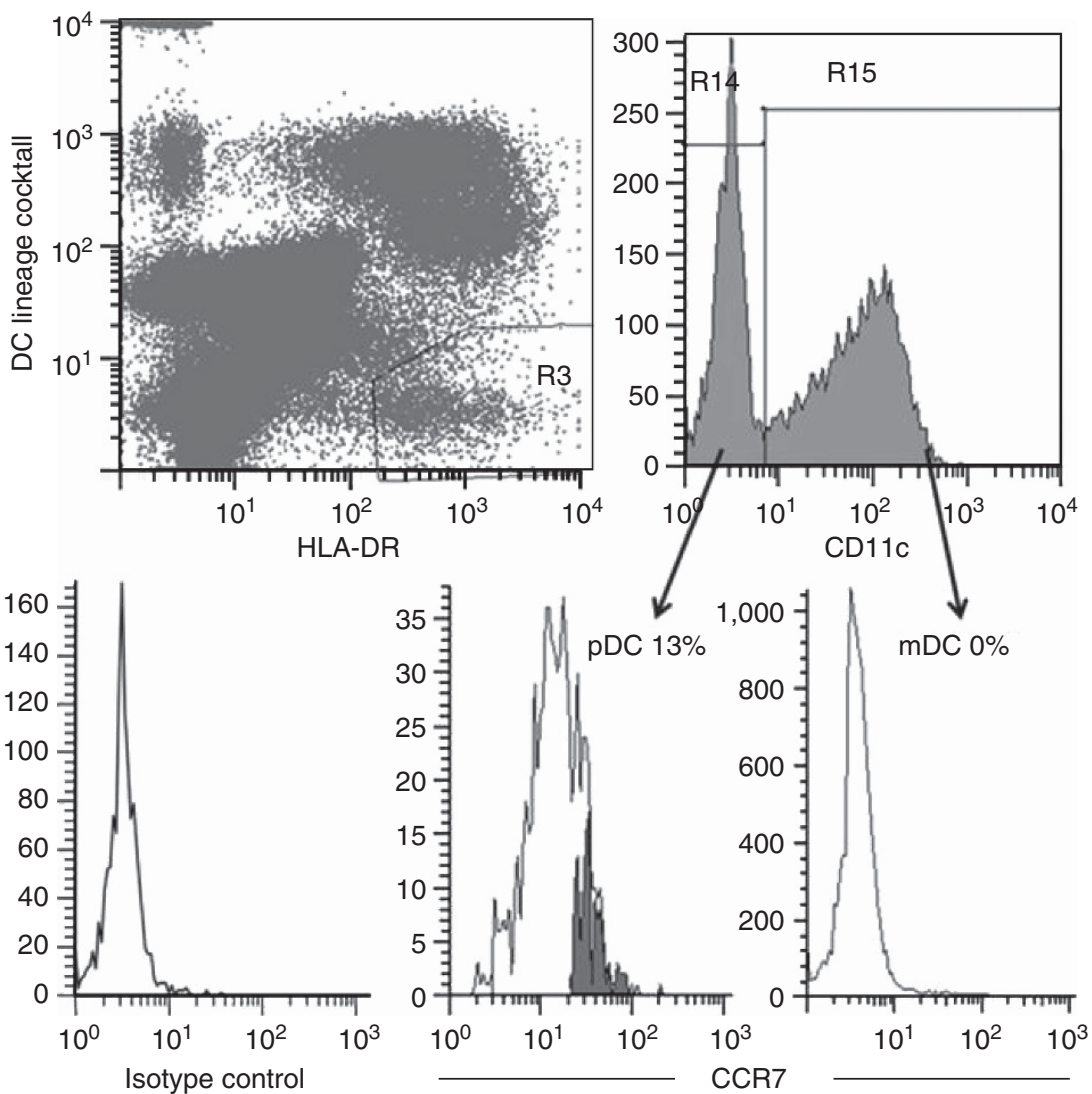

Figure 1 Expression of C-C motif chemokine receptor 7 (CCR7) on freshly isolated blood dendritic cells (DC) subsets. Two DC subsets were identified in blood among the lineage-negative, DR-positive DC: CD11c + myeloid DC (mDC) and CD11c - plasmacytoid DC (pDC). CCR7 was then examined in a fourth channel. The shaded areas of histograms represent the fraction of CCR7 + DC after subtraction of staining from isotype-matched control $(n=8)$ using WinList algorithm (Verity Software, MA).
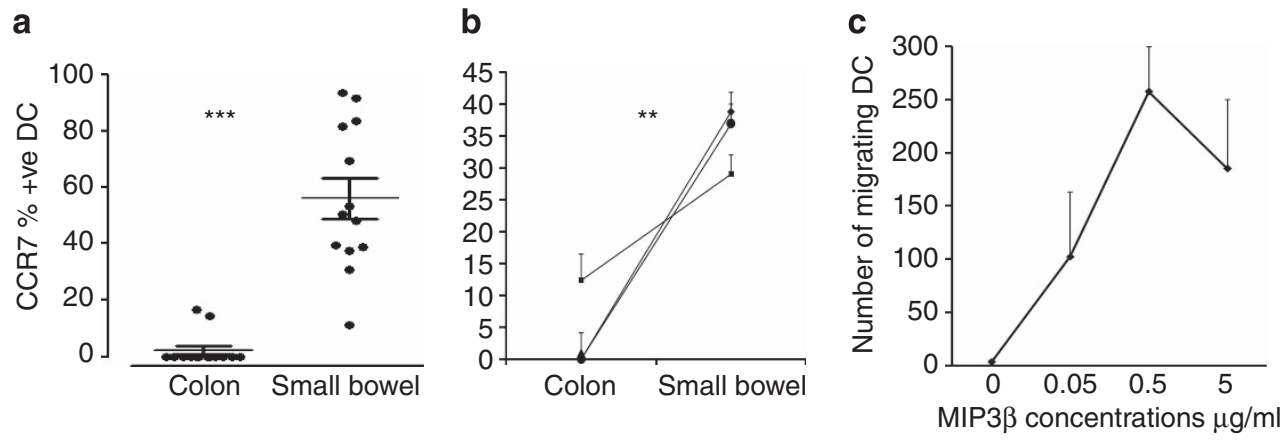

Figure 2 Expression of C-C motif chemokine receptor 7 (CCR7) by intestinal dendritic cells (DC). (a) Terminal ileum DC from normal individuals ( $n=13$ ) expressed significantly higher CCR7 than DC from normal colonic tissues $(n=14)$. (b) DC from normal ileum expressed significantly more CCR7 than that from the normal colon in paired samples from the same individuals $(n=4)$. (c) Migration of CCR7 + DC towards different concentrations of MIP-3 $\beta$ (macrophage inflammatory protein-3 $\beta ; n=4$ ). The statistical significance of differences between groups was determined using unpaired $t$-test for comparison in a and paired $t$-tests for comparisons in $\mathbf{b}$ and $\mathbf{c}$. $P<0.05$ was regarded as significant.

ongoing LepRb receptor production in the normal colonic DC. By contrast, a greater production of LepRb was observed in the normal ileum using paired samples (colonic and terminal ileum biopsies) from the same individuals $(P<005$; Figure 3a).

\section{Expression of pSTAT3 in LepRb-producing DC}

We investigated whether LepRb-producing DC expressed pSTAT3 by assessing the co-expression of both proteins using flow cytometry. We observed significantly higher intracellular expression of pSTAT3 on LepRb-producing DC from the normal ileum than from the colonic DC (Figure 3b).

\section{Changes in CCR7 and LepRb in DC in CD}

To assess whether changes in LepRb and the CCR7 migration marker were relevant to inflammation in $\mathrm{CD}$, we studied both receptors in DC from the blood and gut (both ileum and colon). 

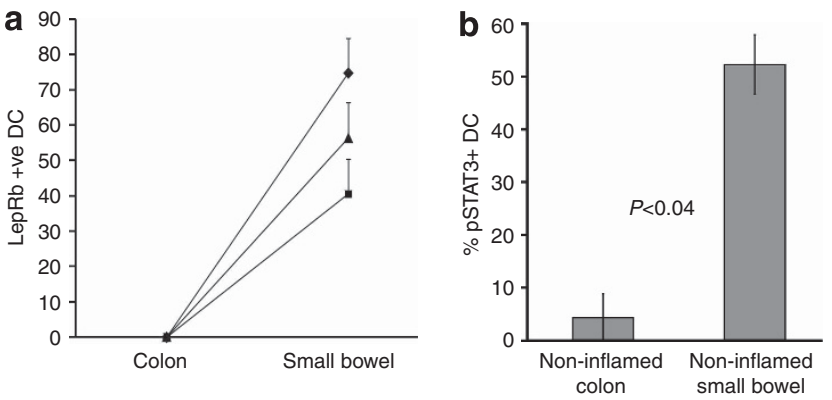

Figure 3 Ongoing production of LepRb (long isoform of leptin receptor) by dendritic cells (DC) from normal blood and tissue. (a) Production of LepRb on normal colonic tissue and normal ileum in paired samples from the same individuals. (b) Expression of phosphorylation of signal transducer and activator of transcription factor 3 (pSTAT3). Numbers shown are the mean percentage of DC positive for LepRb \pm s.e.m. ${ }^{* *} P<0.005$ compared with normal colonic tissue.

In marked contrast to the low levels of expression of CCR7 on normal blood and colonic DC, both blood and colonic DC from CD patients expressed CCR7. In the inflamed colon, CCR7 levels on DC were significantly higher than those on normal colonic DC $(P<0.001$; Figure 4a). Similarly, blood DC from patients with CD expressed significantly higher levels of CCR7 than those from normal individuals $(P<0.0001$; Figure $4 \mathbf{b})$. DC from the colon and blood in CD were also producing LepRb. Thus, LepRb production was significantly increased in DC from colonic biopsies from patients with $\mathrm{CD}$ compared with the minimal-to-undetectable production in DC from control colonic biopsies $(P<0.0001$; Figure 4c) and also significantly increased in DC from peripheral blood mononuclear cells (PBMC) of CD patients compared with controls $(P<0.0001$; Figure $4 d)$. Because of the similarity in LepRb production and CCR7 expression on gut DC, we investigated their distribution in colonic tissue sections from $\mathrm{CD}$ patients. Immunofluorescence microscopy revealed that LepRb expression (green color) was abundant in the cytoplasm of the epithelial cells and CCR7 positive cells (red color) were scattered in the cytoplasm of the lamina propria cells. Colocalization between LepRb and CCR7 was observed mainly in the lamina propria (amber color, Figure 4e). Real-time PCR methods also confirmed that LepRb mRNA levels were significantly higher in enriched blood DC obtained from patients with $\mathrm{CD}$ than from normal controls $(P<0.008$; Figure 4f).

\section{Expression of costimulatory/maturation molecules by freshly isolated DC from the ileum and colon}

To assess whether differences in expression of CCR7 and LepRb were related to maturation status of DC in different gut compartments, we checked the maturation status of DC in the ileum and colon by comparing expression of costimulation and maturation markers in freshly isolated cells and after overnight culture, which is known to cause maturation of DC. ${ }^{25}$

Expression of costimulatory markers CD40, CD80, and CD86 on DC from normal ileum and colon was compared. The expression levels of these costimulatory molecules were low and comparable between DC from both intestinal compartments indicating an immature phenotype (Figure 5a), in contrast with the higher CD80 levels following overnight culture (Figure 5b).

We confirmed the immature phenotype of these DC in both normal and inflamed colonic tissues by immunohistochemistry and electron microscopy. Immunohistochemical investigations showed that DC positive for DC-SIGN, a marker for immature DC, were abundant in normal tissue and scattered evenly in the lamina propria. However, in the inflamed tissue, DC-SIGNpositive DC were significantly more abundant and located mainly below the epithelium (Figure 6a and b). By contrast, DC positive for DC-LAMP, a marker for mature DC, were few and scattered evenly in the lamina propria of both types of tissues (Figure $6 \mathrm{c}$ and d). Moreover, when labeled cells were counted, the absolute numbers of DC-SIGN-positive DC were significantly increased in colonic tissue from $\mathrm{CD}$ patients compared with healthy controls. By contrast, there were no significant differences between inflamed and normal colonic tissues in the expression of DC-LAMP (Figure $\mathbf{6 e}$ and $\mathbf{f}$ ).

Likewise, electron microscopy showed that in both types of tissue the majority of DC were small cells with short veils relative to their size, small cytoplasmic area, and heterochromatic nuclei with thick chromatin layer surrounding the nucleus with chromatic dense clusters within the nucleus, indicating that these cells are type 1 immature $\mathrm{DC}^{26}$ (Figure 6g and $\mathbf{h}$ ).

Collectively, these findings suggest that, in both normal and inflamed tissues, immature DC are predominant and, therefore, the differences in expression of the CCR7 and LepRb were not related to a different maturation status of the DC from the two gut compartments.

\section{A mechanistic role for leptin in CCR7 induction}

When incubated with leptin for $4 \mathrm{~h}$, the expression of CCR7 on normal colonic DC was significantly increased $(P<0.01$; Figure 7a). Chemotaxis assays demonstrated the functionality of CCR7 by a concentration-dependent increase in the migration of DC towards MIP-3 $\beta$. By contrast, low DC migration was observed in non-leptinstimulated lamina propria mononuclear cells (LPMC) (Figure 7b).

\section{Effects of CD colonic microenvironment on CCR7 expression and ongoing lepRb production in blood DC}

To further assess leptin-dependent CCR7 induction, we investigated the effect of culture supernatant of biopsies from CD patients CD-SN (Crohn's disease supernatant-conditioned DC). Supernatants increased the expression of CCR7 on blood DC, and this increase was significantly blocked by monoclonal antibody against leptin receptor (Figure 8a and b).

\section{DISCUSSION}

The current study provides evidence that leptin stimulates DC migration, via the long, signaling form of its receptor LepRb, to induce functional CCR7 expression constitutively in the normal human ileum, a mechanism absent in both the normal 

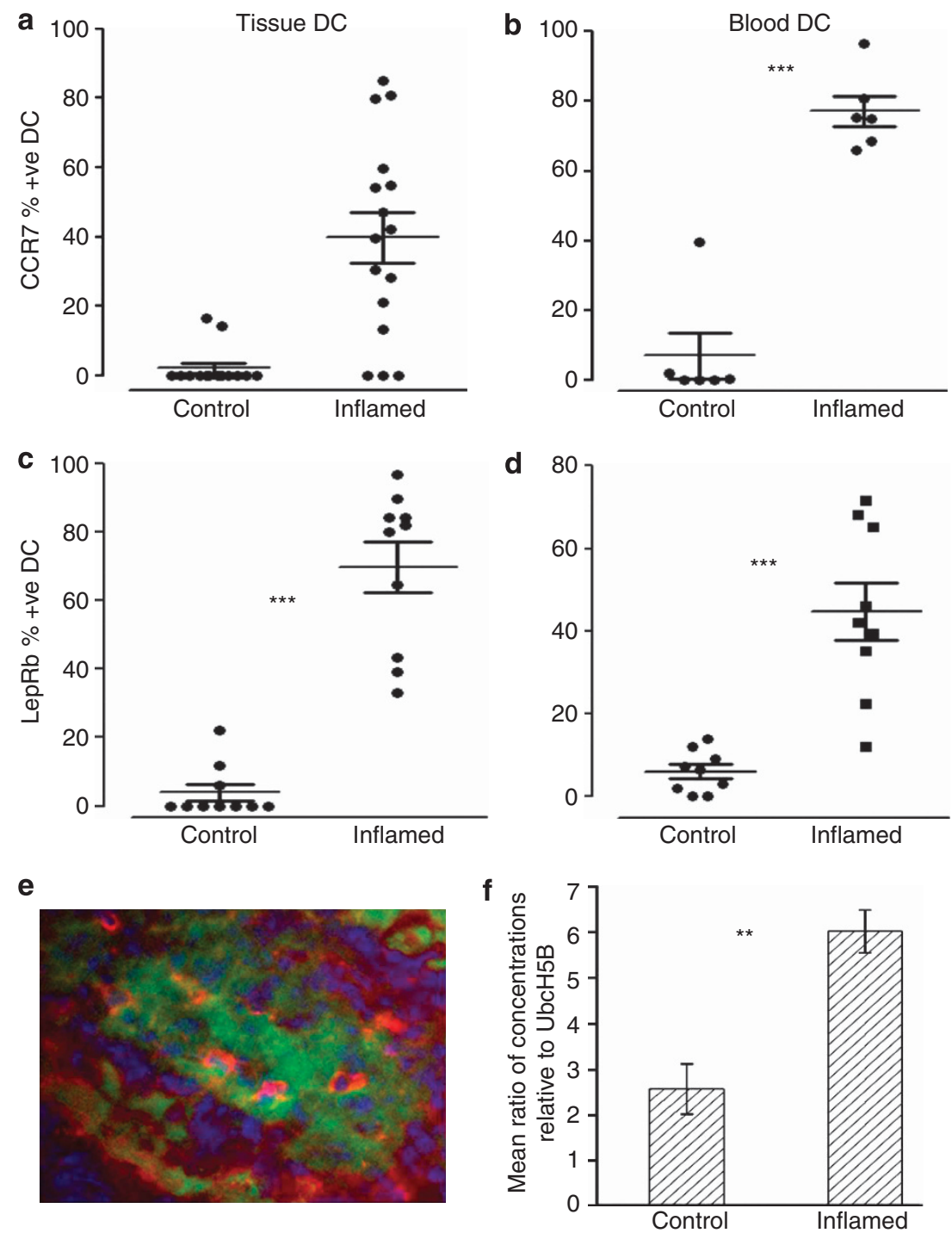

Figure 4 Ongoing production of LepRb (long isoform of leptin receptor) and C-C motif chemokine receptor 7 (CCR7) expression on dendritic cells (DC) from normal individuals and Crohn's disease (CD) patients. (a) CCR7 expression on colonic DC from normal and CD tissue ( $n=14)$. (b) CCR7 expression on DC from normal and CD PBMC $(n=6)$. (c) Ongoing production of LepRb on colonic DC from normal and CD tissues $(n=10)$. (d) Ongoing production of LepRb on DC from normal and CD peripheral blood mononuclear cells $(n=9)$. (e) Double immunofluorescence staining of LepRb (green color), CCR7 (red color) or co-localization (amber color) in the lamina propria of CD colonic tissue. (f) Expression of LepRb mRNA in DC enriched from blood of patients with $\mathrm{CD}$ and normal individuals using real-time reverse transcriptase-PCR $(n=3) .{ }^{* *} P<0.001$ and ${ }^{* * *} P<0.0001$ compared with colonic DC from healthy controls.

colon and, systemically in $\mathrm{mDC}$, in blood. By contrast, the presence of high levels of both LepRb and functional CCR7 in DC from the colon and blood in CD suggests a role for this system in the development of inflammation; in normal colon and blood DC, leptin promoted CCR7 expression and the capacity of DC to migrate towards MIP- $3 \beta$, a lymph node chemokine, as previously reported in blood DC. ${ }^{20} \mathrm{~A}$ role for leptin in CD is likely, as mesenteric hypertrophy of fat occurs in early $\mathrm{CD},{ }^{27}$ accompanied by local increase in both the adipokine leptin mRNA and protein. ${ }^{9}$ Involvement of leptin in the colon in $\mathrm{CD}$ was further indicated by the upregulation of CCR7 on DC by CD-SN, an effect blocked by antibody to LepRb.
DC maturation is characterized by increases in surface expression of co-stimulatory and adhesion molecules and chemokines receptors, as well as secretion of cytokines, chemokines and proteases. ${ }^{28}$ Flow cytometry and in situ phenotyping of DC confirmed that, as in the normal colon, immature DC were predominant in normal ileum, suggesting that maturation induced by inflammation is not a prerequisite for CCR7 and LepRb expression in ileum.

Although there is controversy in the literature, ${ }^{29}$ our data indicate that DC from inflamed colonic tissue are also predominantly immature, as determined by DC-SIGN and DC-LAMP expression. Electron micrographs also confirmed that the majority of DC in inflamed tissue were of the immature 



Figure 5 Expression of maturation markers by colonic and ileal dendritic cells (DC). (a) Colonic and ileal DC have a similar immature phenotype with low levels of CD40, CD83, and CD86. (b) Expression of CD80 by freshly isolated and matured DC from colon and ileum; colonic and ileal DC expressed similar low levels of CD80 (day 0). Expression of CD80 had increased after culture for overnight (day 1). The histograms are of antibody-stained samples. The shaded areas represent the fraction of positive staining cells after subtraction of staining from isotype-matched control. The percentage values represent the positive proportion of cells. Numbers in brackets are intensity ratios of stained cells compared with those in the isotype control $(n=8)$.

phenotype, in line with observations in murine models. ${ }^{30}$ These in situ results also confirm that the increase in CCR7 expression on colonic DC is independent of maturation during inflammation. It is also possible that CCR7 is upregulated before full maturation and exit of DC to lymph nodes.

There is an emerging consensus that DC maturation is not an all-or-nothing phenomenon. For instance, when immature DC were transfected with CCR7 in vitro, they acquired low levels of co-stimulatory molecules, their migration capacity in response to MIP-3 $\beta$ significantly increased, but their ability to stimulate T cells was impaired, ${ }^{31}$ suggesting a semi-mature phenotype of DC. ${ }^{32,33}$ Consistent with this notion, the lower expression of costimulatory molecules on fresh normal ileum and colonic DC compared with those induced on maturation by overnight culture suggest that the CCR7-positive DC were of immature or semi-mature phenotype; such immature cells would function to induce T-cell anergy and, therefore, suppress T-cell-mediated immune responses. ${ }^{34,35}$

The gut mucosa is constantly exposed to both commensal and pathogenic foreign antigens, and DC are instrumental in maintaining gut immune homeostasis. In response to pathogenic antigens, DC initiate an immune response by processing these antigens, maturing and migrating to local lymph nodes, and stimulating T cells. DC sample antigens from the lumen, either directly by passing dendrites through the epithelium ${ }^{36}$ or indirectly by sampling antigen that is transported across epithelial cells or captured by M-cells in the Peyer's patches. The small intestine is the section of the gut where nutrients are 
a
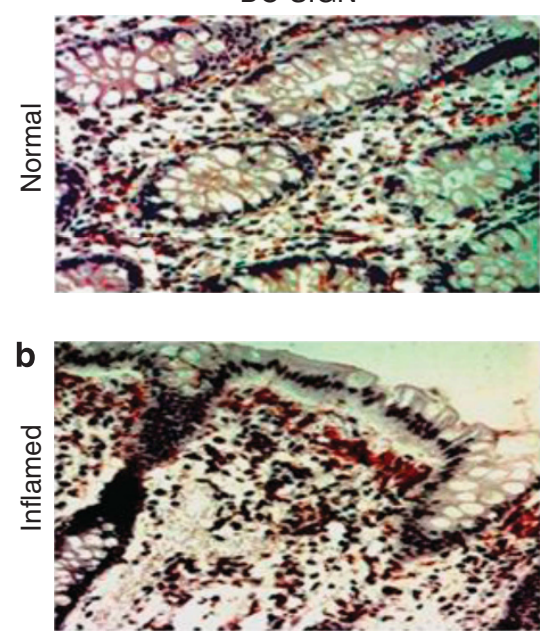

e

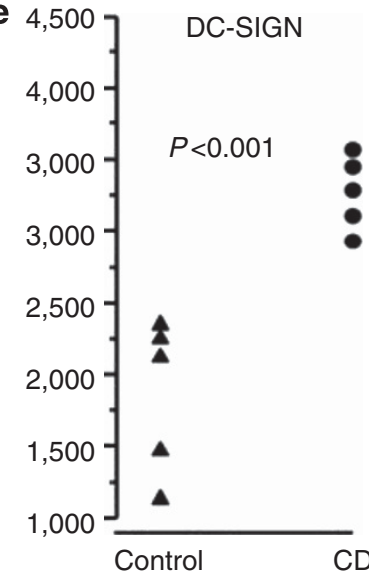

g



C
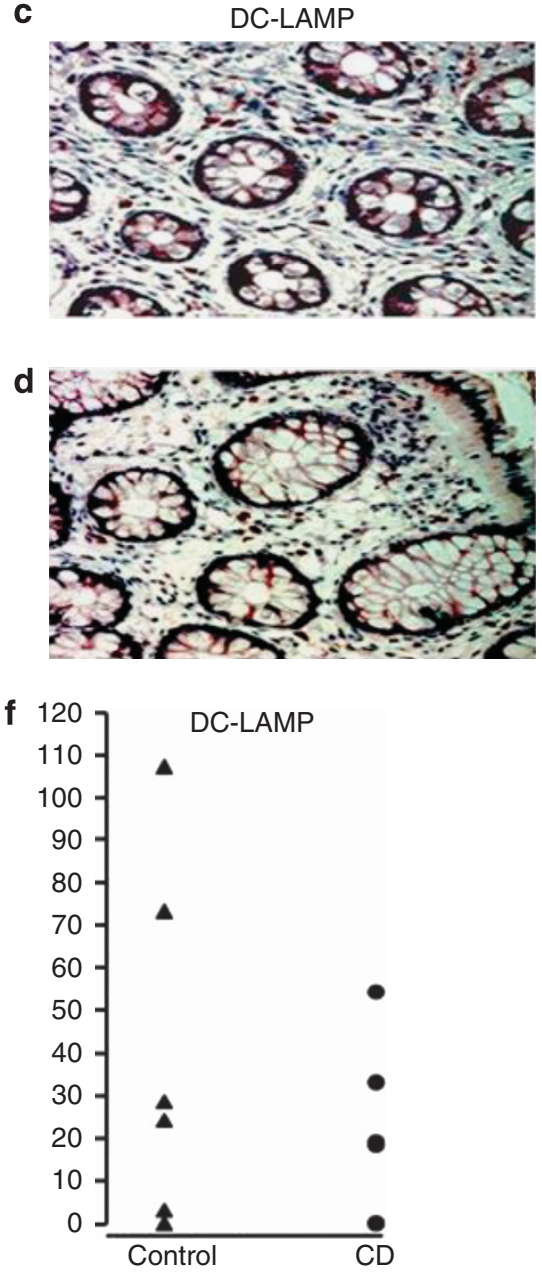

h

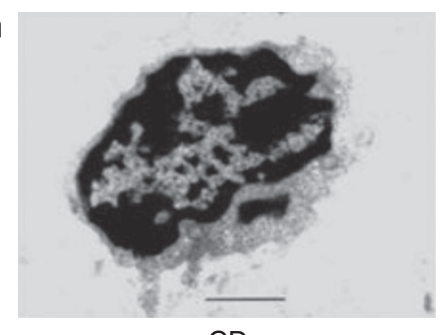

CD

Figure 6 Localization and numbers of DC-SIGN (a marker for immature dendritic cells (DC)) and DC-LAMP (a marker for mature DC) in normal and inflammatory bowel disease colonic mucosae by immunohistochemistry. DC-SIGN ${ }^{+}$immature cells were scattered evenly throughout the mucosa in both (a) healthy controls and (b) Crohn's disease (CD) tissues, with a tendency to cluster below the epithelium in CD tissues. DC-LAMP + mature DC were very few in number in both (c) control and (d) inflamed tissues and were scattered in the lamina propria in all the tissue samples. (e) DC-SIGN + DC were significantly higher in CD tissues compared with controls. (f) DC-LAMP + DC numbers were similar in both types of tissue. Electron micrographs of DC from the lamina propria showing type-1, myeloid immature DC in (g) normal and (h) CD colonic tissues $(\mathrm{bar}=1 \mu \mathrm{m})$.

absorbed, and due to high flow rate and bile acid, the microflora concentration is relatively small compared with that in the large intestine. Therefore, the differential expression of LepRb and CCR7 on DC from the small and large intestine could be due to the different microenvironments in the two compartments. It is likely that DC in healthy small bowel traffic constitutively to draining lymph nodes to transport self-antigen, e.g., from apoptotic cells, to induce oral tolerance, possibly, by promoting tolerogenic naive T-cell profiles. ${ }^{37}$ Conversely, in the large intestine, tolerance is maintained by the lack of DC migration; migration of DC with upregulation of CCR7 in this compartment may only occur upon encountering danger signals, to induce immunogenic responses.

Our data have demonstrated a mechanistic role for leptin as shown by the significant increase of CCR7 expression on normal colonic DC when incubated with leptin. This upregulation of CCR7 was functional as demonstrated by the concentration-dependent migration of DC towards the 
chemoattractant MIP-3 $\beta$. CCR7-dependent migration requires the JAK/STAT pathway as mature DC migration towards MIP$3 \beta$ and CCL20 in Jak3 ${ }^{-1-}$ mice was impaired. ${ }^{38}$ Leptin shares the same pathway but also activates the transcriptional factor nuclear factor- $\mathrm{\kappa B}$, which mediates the upregulation of CCR7 expression..$^{39,40}$ This activity may explain the effect of leptin on CCR7 expression in DC from normal gut biopsies where the production of LepRb was initially low. Indeed, leptin signaling via LepRb also involves Src homology phosphatase 2 and STAT5 among other downstream cascade of events. ${ }^{15}$ However, in this study, we focused on STAT3 known to be strongly tyrosin phosphorylated in $\mathrm{IBD}^{41}$ and a mediator of inflammation in IBD and other forms of gut inflammation. ${ }^{42}$

Information on leptin concentrations in the serum of $\mathrm{CD}$ patients is contradictory; while some reported no correlation between plasma concentrations and the degree of inflammation in patients with $\mathrm{IBD},{ }^{43}$ others have shown decrease in leptin levels in the serum of IBD patients. ${ }^{44}$ These observations suggest that the serum levels of leptin in IBD patients may be affected by factors such as generalized loss of subcutaneous fat and may not reflect increases in "local" mesenteric leptin mRNA and protein levels in patients with IBD; $;^{45}$ patients with $\mathrm{CD}$ are often thin but have fat mesentery. This anomaly could
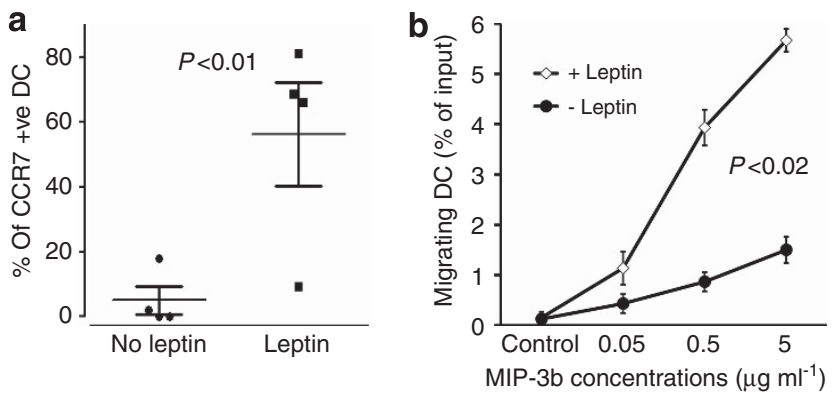

Figure 7 Effect of leptin on functional C-C motif chemokine receptor 7 (CCR7) expression on dendritic cells (DC) from the large intestine. (a) DC from leptin-treated lamina propria mononuclear cells (LPMC) expressed significantly higher levels of CCR7 expression compared with untreated LPMC $(n=4)$. (b) Migration of leptin-stimulated CCR7-positive DC towards different concentrations of MIP-3 $\beta$ (macrophage inflammatory protein-3 $\beta ; n=4)$. be due to an endocrine or paracrine feed-back effect, from the production of other adipokines that inhibit the leptin production by adipocytes and other immune cells. For example, resistin expressed by macrophages and circulating mononulclear cells increases the production of TNF- $\alpha$ and IL- $6 .{ }^{46}$ TNF$\alpha$ subsequently downregulates the production of leptin by adipocytes. ${ }^{47}$ It would be interesting to investigate whether IBD patients with increased levels of leptin in the plasma have low levels of TNF- $\alpha$.

In conclusion, the coincident increase in CCR7 expression and LepRb production in DC from inflamed tissues and upregulation of CCR7 on DC following culture with leptin, suggest that leptin has a role in regulating CCR7 and, hence, in DC migration from the gut as part of its role in inflammation in CD. Our results suggest that leptin/leptin receptors could be immunomodulatory therapeutic targets for the treatment of $\mathrm{CD}$; blocking of the upregulated leptin/CCR7 pathway in colonic and systemic DC in CD might be invoked therapeutically.

\section{METHODS}

Blood and tissues. The study was approved by the Outer West London Regional Ethics Committee. All patients provided written informed consent on entry to the study. Venous blood was obtained from healthy volunteers and collected into heparinised Vacutainers (Becton Dickinson, Oxford, UK). Intestinal tissue was obtained during routine endoscopy or from surgical resections. Non-inflamed tissue was obtained from patients with macroscopically and histologically normal intestine who had been referred with rectal bleeding or change in bowel habit (endoscopic samples) and from patients at risk of cancer. Inflamed tissue was obtained from patients with IBD. Informed consent was obtained from all patients and the local ethical committee approved the protocols.

Antibodies. Antibodies to HLA-DR (G46-6), CD80 (L307.4), CD83 (HB15e), CD34 (581), CCR7 (2H4), pSTAT3 (4/pSTAT3) and CD8 (SK1-RPA-T8) and maching isotype control were purchased from BD Pharmingen, Oxford, UK. Antibody to LepRb (Ob-Rb-COOH) and (C20) were from Santa Cruz Biotechnology, CA, USA. Antibodies to CD3 (UCHT-1), CD14 (MIP9), CD16 (B73.1), CD19 (4G7), and CD56 (N901) came from Beckman Coulter, High Wycombe, UK. Antibodies to CD14 (TÜK-4) and CD19 (SJ25-C1) were obtained from ABD Serotec, Kidlington, UK. Anti-CD11c (clone KB90) was purchased from Dako - Alere, Stockport, UK. Biotinylated anti-mouse
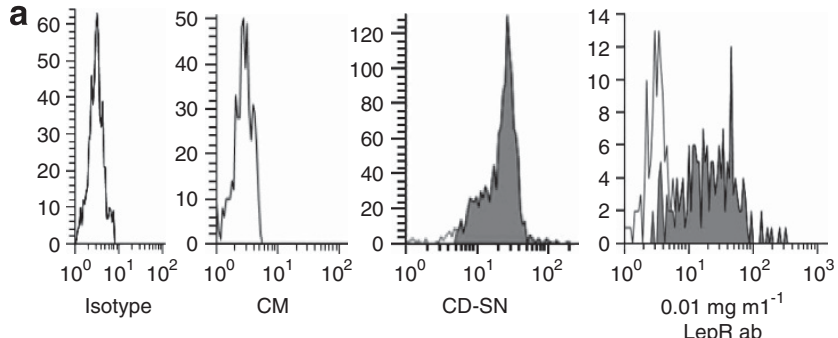


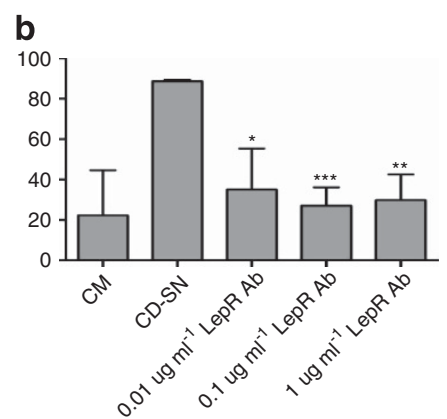

Figure 8 Effects of microenvironment of Crohn's disease (CD) colonic tissue on C-C motif chemokine receptor 7 (CCR7) expression. (a) Representative experiment showing a profile of CCR7 expression after incubation with antibody (ab) against leptin receptor (LepR). (b) Data are the mean \pm s.e. $(n=4)$. ${ }^{\star} P<0.02,{ }^{\star \star} P<0.003$, and ${ }^{\star * \star} P<0.0005$ compared with CD-SN (Crohn's disease supernatant-Conditioned DC). CM, complete medium. 
IgM (R6-60.2) and phycoerythrin-conjugated streptavidin obtained from BD Pharmingen. Anti-DC-SIGN/CD209-phycoerythrin, (120507) was from R\&D Systems, Abingdon, UK, and DC-LAMP/CD208 (104.G4) from Beckman Coulter. Appropriate isotype-matched control antibodies were purchased from the same companies.

Blood processing and maturation of DC. PBMC were obtained by centrifugation over Ficoll-Paque-Plus (Amersham Biosciences, Little Chalfont, UK) at $650 \mathrm{~g}$, for $30 \mathrm{~min}$ at room temperature. To generate mature blood DC, PBMC were cultured overnight $\left(37^{\circ} \mathrm{C}\right.$ in humidified $5 \% \mathrm{CO}_{2}$ ) at $20 \times 10^{6} / 5 \mathrm{ml}$ in complete medium (Dutch-modified RPMI 1640 (Sigma-Aldrich, Dorset, UK) containing $100 \mu \mathrm{ml}^{-1}$ penicillin/streptomycin, $2 \mathrm{~mm}$ L-glutamine, $50 \mu \mathrm{g} \mathrm{ml}^{-1}$ gentamicine (Sigma-Aldrich), and 10\% fetal calf serum (TCS cellworks, Buckingham, UK)) in $25 \mathrm{~cm}^{2}$ tissue culture flasks (Becton Dickinson, Oxford, UK). The cells were cultured overnight at $37^{\circ} \mathrm{C}$ in humidified $5 \% \mathrm{CO}_{2}$ and non-adherent cells recovered by centrifugation and DC were identified by flow cytometery.

Isolation of LPMC. To obtain a fresh DC-containing LPMC population with the minimal manipulation, a rapid collagenase digestion method was used. ${ }^{25}$ To remove mucus, tissue was incubated at room temperature for $20 \mathrm{~min}$, with occasional agitation, in calcium- and magnesium-free HBSS (Hank's Balanced Salt Solution; Life Technologies, Paisley, UK) containing $1 \mathrm{~mm}$ dithiothreitol (Sigma-Aldrich, Dorset, UK). Tissue was then blotted and transferred to HBSS containing $1 \mathrm{~mm}$ EDTA for removal of the epithelium. After incubation at $37^{\circ} \mathrm{C}$ for $30 \mathrm{~min}$ with gentle shaking, the tissue was washed in HBSS repeatedly until the supernatant was macroscopically free of released epithelial cells. Tissue denuded of epithelial cells was incubated with gentle shaking at $37^{\circ} \mathrm{C}$ in HEPES (4-(2-hydroxyethyl)1-piperazineethanesulfonic acid)-buffered RPMI-1640 (SigmaAldrich) containing $1 \mathrm{mg} \mathrm{ml}^{-1}$ collagenase D, $20 \mathrm{mg} \mathrm{ml}^{-1}$ DNase I (Roche, Burgess Hill, UK) and 2\% fetal calf serum. Incubation was stopped when visual inspection revealed complete digestion of the tissue, typically after $90-120 \mathrm{~min}$. Mononuclear cells were then separated $(650 \mathrm{~g}, 20 \mathrm{~min}$, room temperature.) on Ficoll-Paque (Amersham Biosciences, Little Chalfont, UK) and washed in complete medium.

Cell surface labeling. For whole-blood staining, antibodies were added to $100 \mu \mathrm{l}$ of blood at predetermined optimal concentrations and incubated at room temperature for 15 mins. Cells from intestinal tissues were antibody-labeled in $100 \mu \mathrm{l} \mathrm{FACS}$ buffer on ice for $20 \mathrm{~min}$ in the presence of $15 \%$ fetal calf serum to reduce non-specific binding. To determine the effect of leptin on CCR7 expression on blood and tissue DC, leptin was incubated with PBMC or LPMC for $4-6 \mathrm{~h}$ at a concentration of $20 \mathrm{~nm}$. CCR7 expression on DC was then determined using multi-color flow cytometer (FACSCalibur, BD, UK).

Intracellular labeling. The production of LepRb by Blood DC and LPDC was determined as previously discribed. ${ }^{48}$ Briefly, LPMC and PBMC were placed in wells at $5 \times 10^{5}$ cells per well in 1 -ml complete medium in Falcon 24-well tissue culture plates (Becton Dickinson). LPMC were incubated with and without monensin $\left(3 \mathrm{mmoll}^{-1}\right)$ for $4 \mathrm{~h}$ at $37^{\circ} \mathrm{C}$ in a humidified atmosphere of $5 \% \mathrm{CO}_{2}$ in air. Monensin leads to the accumulation of protiens at the Golgi apparatus. Cultured cells were then washed, fixed with Leucoperm A $(100 \mathrm{~mL})$, and permeabilized with Leucoperm B $(50 \mathrm{ml}$; ABD Serotec, Kidlington, UK). Cells were then incubated with anti-LepRb, pSTAT3, or isotype control (IgG2a-phycoerythrin for $30 \mathrm{~min}$, washed, then incubated with antimouse FITC-conjugated $\operatorname{IgG}$ for $20 \mathrm{~min}$, washed and finally resuspended in $1 \%$ paraformaldehyde. By using the monensin method and applying the same antibody to stain both samples in a comparison, the potential problems associated with differences between antibodies with regard to non-specific binding to permeablised cells (for instance, due to variations in free fluorochrome content) were avoided; the technique was thus very sensitive. The results are presented graphically as a histogram of the staining in the presence of monensin in which a shaded area represents the fraction of positive cells after subtraction of the no monensin labeling. The differences between the paired histograms were determined as shown below.

Flow cytometry and data analysis. Data were acquired on a fourcolor FACScalibur machine (Becton Dickinson). List mode data files were analyzed using WinList-5.0 software (Verity, Topsham, ME). DC were identified as HLA-DR + Lin - cells where Lin is a mixture of monoclonal antibodies to CD3, CD14, CD16, CD19 CD34, and CD56. The $\mathrm{mDC}$ was identified as a CD11c + subset; plasmacytoid DC were CD11c - . To determine expression of other markers, single parameter histogram plots were prepared for gated DC populations. Histograms of isotype control labeling were subtracted from histograms of specific antibody staining using the Super-enhanced $D_{\max }$ normalized subtraction (SED) facility in WinList. The statistical significance of differences between the two curves were assessed by Kolmogorov and Smirnov (K-S) statistics according to the formula: ${ }^{49}$

$$
D_{\text {crit }}=D_{\max } / \sqrt{\{(n 1+n 2) /(n 1 x n 2)\}}
$$

where $D_{\max }$ is the maximum difference between cumulative normalized histograms of isotype control and antibody labeling, $n 1$ is the number of events in the antibody-labeled sample, and $n 2$ is the number of events in the isotype-matched control sample.

Samples with an insignificant $D_{\text {crit }}$ value $(P>0.05, \mathrm{~K}-\mathrm{S}$ statistics), or where the subtraction program suggests a negative value (i.e., there is greater labeling with the isotype control), were regarded as zero. Results are presented as the percentage of positive cells after subtraction of the isotype control. The statistical significance of differences between experimental groups was determined using $t$-tests and $P<0.05$ was regarded as significant.

Chemotaxis assay. Chemotactic responses to chemokines were analyzed in a $5-\mu \mathrm{m}$ Transwell system (Corning, Lowell, MA) using a membrane pore size of $5-\mu \mathrm{m}$. In a volume of $100 \mu \mathrm{l}, 5 \times 10^{5} \mathrm{PBMC}$ or LPMC were placed in the upper chamber of the Transwell. Into the bottom chamber was placed $600 \mu \mathrm{l}$ at concentrations of $0.05 \mathrm{mg} \mathrm{ml}^{-1}$, $0.5 \mathrm{mg} \mathrm{ml}^{-1}$ and $5 \mathrm{mg} \mathrm{ml}^{-1}$ of the chemokine in complete medium. Control wells contained medium alone. Plates were incubated for 90 min at $37^{\circ} \mathrm{C}$ in humidified $5 \% \mathrm{CO}_{2}$ and migrated cells recovered by centrifugation. The number of migrating cells was determined by flow cytometry using flow count fluorospheres (Beckman Coulter, High Wycombe, UK) to determine cell numbers. DC were detected by antibody labeling as described above.

Enrichment of DC from PBMC. DC-enriched (LDC (low-density cells)) were generated as previously described ${ }^{50}$ and consistently gave a yield of $5-7 \%$ of the total number of PBMC isolated from all donors. Briefly, PBMC were incubated for $24 \mathrm{~h}$ (at $37^{\circ} \mathrm{C}, 5 \% \mathrm{CO}_{2}$, high humidity) in complete medium (Dutch-modified RPMI 1640 (SigmaAldrich) containing $100 \mu \mathrm{ml}^{-1}$ penicillin/streptomycin, $2 \mathrm{~mm}$ L-glutamine, and $10 \%$ fetal calf serum (TCS cellworks, Buckingham, UK) at $4 \times 10^{6} \mathrm{ml}^{-1}$ in T25 tissue culture flasks. Non-adherent cells were centrifuged $(600 \mathrm{~g}, 15 \mathrm{~min}$ at room temperature) over NycoPrep (Progen Biotechnik, Heidelberg, Germany), and LDC recovered from the interface, washed, counted and lysed in $1 \mathrm{ml}$ lysis buffer (Miltenyi Biotec, Bisley, UK) and stored at $-80^{\circ} \mathrm{C}$.

DC conditioning. Colonic biopsies from four CD patients were collected in ice-chilled complete medium and cultured within $1 \mathrm{~h}$ in complete medium in 24-well culture dishes ( 1 biopsy per ml per well) for $24 \mathrm{~h}\left(37^{\circ} \mathrm{C}, 5 \% \mathrm{CO}_{2}\right.$, high humidity). In addition, $1 \mathrm{ml}$ of complete medium was added to empty wells and incubated for use as a control. Media were centrifuged (1500 r.p.m., $t=5^{\prime}$ ), and supernatants used to condition freshly isolated PBMC. PBMC were incubated at (1 million cells $\mathrm{ml}^{-1}$ ) in the culture medium from colon biopsies or control. Conditioned PBMC were incubated with anti-lepRb at concentrations 
of $0.01 \mu \mathrm{g} \mathrm{ml}^{-1}, 0.1 \mu \mathrm{g} \mathrm{ml}^{-1}$, and $1 \mu \mathrm{g} \mathrm{ml}^{-1}$ for $4 \mathrm{~h}$. DC were identified as described above and CCR7 on DC was analyzed.

Semi-quantitative reverse transcriptase PCR. The lysed cells were thawed and RNA was isolated using the $\mu$ macs small-scale mRNA isolation kit (Miltenyi Biotec, UK). Reverse transcription was performed using the Invitrogen Cloned AMV First Strand cDNA Synthesis Kit (Invitrogen, Paisley, UK).

The primers were obtained from Invitrogen and sequences were as follows:

The primers were designed so that the regions amplified would cross exons and therefore any genomic DNA would not be amplified during PCR. Blast searches were performed to ensure that the amplified sequences only corresponded to the desired genes. The primers were obtained from Invitrogen Life Technologies and sequences were as follows: LepRb: forward, 5'-GTTCCGAACCCCAAGA-3', reverse, $5^{\prime}$-CCACAGTTGTTGGCAT-3'; and UbcH5B (housekeeping gene): forward, (5'-AGTGTTCAGCAGGTCC-3', reverse, 5' ${ }^{\prime}$-TCCGAGCAATCTCAGG- $3^{\prime}$. Real-time reverse transcriptase-PCR was used using the FastStart SYBR Green method with the Roche Light Cycler (Roche Applied Science, Burgess Hill, UK) following the manufacturer's protocol and using the following conditions: $95^{\circ} \mathrm{C}$ for $10 \mathrm{~min} 1$ cycle; $95^{\circ} \mathrm{C}$ for $10 \mathrm{~s} ; 60^{\circ} \mathrm{C}$ for $5 \mathrm{~s} 45$ cycles; and $72^{\circ} \mathrm{C}$ for $8 \mathrm{~s}$. The relative quantification software was used to quantify the expression of each test gene relative to the housekeeping gene for each sample. This was done with reference to a calibration curve for each gene so that the efficiency of the reaction could be taken into account by the software.

Immunohistochemistry. The avidin-biotin complex technique was used. Briefly, slides of paraffin-embedded tissue sections of colonic biopsies were deparaffinised, rehydrated, and endogenous peroxidase activity was blocked with $0.3 \% \mathrm{H}_{2} \mathrm{O}_{2}$ in $100 \%$ methanol for $30 \mathrm{~min}$. Tissues were then incubated with normal goat serum for $30 \mathrm{~min}$ at room temperature and incubated overnight in a humid atmosphere at $4{ }^{\circ} \mathrm{C}$, with primary antibodies, anti-DC-LAMP (1:50) and anti-DCSIGN (1:200). After washing and incubation with biotinylated goat anti-mouse immunoglobulin for $30 \mathrm{~min}$, sections were washed and incubated with the avidin-biotin-peroxidase complex. Subsequently, sections were washed in tap water, counterstained with haematoxylin, placed in acid alcohol for 3-5 s, and washed in water. Sections were dehydrated, cleared in xylene, and finally mounted with DEPEX. Red-brown staining indicates positive immunoreactivity. Control sections were incubated with normal serum instead of primary antibodies. All incubation times were similar and reactions were stopped simultaneously on sections to standardize the quantitative methods.

For quantification of antibody staining, all positive cells in every possible microscopic field in all sections were counted under highpower magnification ( $\times 40$ objective). To enable comparison between sections of different sizes, results were expressed as number of positive cells per 10 microscopic fields. Only morphologically well-defined positively stained cells were considered for analysis. All assessments of immunostaining were independently evaluated by two investigators who had no previous knowledge of the clinical data.

Immunofluorescence microscopy. Double labeling was performed on $4 \mu \mathrm{M}$ frozen section. Sections were fixed in cold acetone, washed with phosphate-buffered saline (PBS), incubated with normal mouse serum (NMS), and incubated with anti-lepRb (1:500) for overnight. After three washes in PBS, sections were incubated with NMS followed by anti-mouse FITC IgG for $3 \mathrm{~h}$. Sections were incubated with NMS followed by anti-CCR7 (R\&D Systems, UK) for $3 \mathrm{~h}$, then washed with PBS, and non-specific binding was blocked with NMS before incubation with TRITC-conjugated secondary antibody (Sigma). Sections were washed and mounted under cover slips with mounting medium (Dako, UK). Control experiments were performed by omitting the primary antibodies.
Electron microscopy. Ultrathin sections of colonic tissues (100-nm thick) were were stained with Reynold's lead citrate, the grid was carbon coated, and visualized using a JEOL 1200 EX electron microscope (JEOL, Tachikawa, Japan). DC were identified as previously described ${ }^{51}$ and have been classified into three types by electron microscopy: Type 1-myeloid iDC,small cells with short veils relative to their size, small cytoplasm area, and heterochromatic nuclei with thick chromatin layer surrounding the nucleus and chromatic dense clusters within the nucleus; Type 2-possible plasmacytoid DC, are bigger than type-1, with fewer projections, thin chromatin layer surrounding the nucleus, and disaggregated chromatin within the nucleus; and Type 3-mature mDC, have long veiled projections.

\section{ACKNOWLEDGEMENTS}

We thank The Broad Medical Research Program of the Broad Foundation, USA, the Medical Research Council Trust, UK, St Mark's Hospital Foundation, UK, the Biotechnology and Biological Research Council Institute Strategic Program for Gut Health and Food Safety BB/J004529/1 and the Brigid Balfour Fund for funding this study. A.I.F.B. is also funded by Diabetes UK and the BRC at the Imperial College Healthcare Trust.

\section{DISCLOSURE}

The authors declared no conflict of interest.

(c) 2013 Society for Mucosal Immunology

\section{REFERENCES}

1. Banchereau, J. et al. Immunobiology of dendritic cells. Annu. Rev. Immunol. 18, 767-811 (2000).

2. Huang, F.P. et al. A discrete subpopulation of dendritic cells transports apoptotic intestinal epithelial cells to $\mathrm{T}$ cell areas of mesenteric lymph nodes. J. Exp. Med. 191, 435-444 (2000).

3. Sallusto, F. et al. Rapid and coordinated switch in chemokine receptor expression during dendritic cell maturation. Eur. J. Immunol. 28, 2760-2769 (1998).

4. Yanagihara, S., Komura, E., Nagafune, J., Watarai, H. \& Yamaguchi, Y. EBI1/CCR7 is a new member of dendritic cell chemokine receptor that is up-regulated upon maturation. J. Immunol. 161, 3096-3102 (1998).

5. Luther, S.A., Tang, H.L., Hyman, P.L., Farr, A.G. \& Cyster, J.G. Coexpression of the chemokines ELC and SLC by T zone stromal cells and deletion of the ELC gene in the plt/plt mouse. Proc. Natl. Acad. Sci. USA 97, 12694-12699 (2000).

6. Jang, M.H. et al. CCR7 is critically important for migration of dendritic cells in intestinal lamina propria to mesenteric lymph nodes. J. Immunol. 176, 803-810 (2006).

7. Scheinecker, C., McHugh, R., Shevach, E.M. \& Germain, R.N. Constitutive presentation of a natural tissue autoantigen exclusively by dendritic cells in the draining lymph node. J. Exp. Med. 196, 1079-1090 (2002).

8. Steinman, R.M. \& Nussenzweig, M.C. Avoiding horror autotoxicus: the importance of dendritic cells in peripheral Tcell tolerance. Proc. Natl. Acad. Sci. USA 99, 351-358 (2002).

9. Paul, G. et al. Profiling adipocytokine secretion from creeping fat in Crohn's disease. Inflamm. Bowel Dis. 12, 471-477 (2006).

10. Peyrin-Biroulet, L. et al. Mesenteric fat as a source of $C$ reactive protein and as a target for bacterial translocation in Crohn's disease. Gut 61, 78-85 (2012).

11. Lord, G.M. et al. Leptin modulates the T-cell immune response and reverses starvation-induced immunosuppression. Nature 394, 897-901 (1998).

12. Mattioli, B., Straface, E., Quaranta, M.G., Giordani, L. \& Viora, M. Leptin promotes differentiation and survival of human dendritic cells and licenses them for Th1 priming. J. Immunol. 174, 6820-6828 (2005).

13. Martin-Romero, C., Santos-Alvarez, J., Goberna, R. \& Sanchez-Margalet, V. Human leptin enhances activation and proliferation of human circulating T Iymphocytes. Cell. Immunol. 199, 15-24 (2000).

14. Procaccini, C., Jirillo, E. \& Matarese, G. Leptin as an immunomodulator. Mol. Aspects Med. 33, 35-45 (2012). 
15. Mackey-Lawrence, N.M. \& Petri, W.A. Jr. Leptin and mucosal immunity. Mucosal Immunol. 5, 472-479 (2012).

16. Tartaglia, L.A. et al. Identification and expression cloning of a leptin receptor, OB-R. Cell 83, 1263-1271 (1995).

17. Baumann, H. et al. The full-length leptin receptor has signaling capabilities of interleukin 6-type cytokine receptors. Proc. Natl. Acad. Sci. USA 93, 8374-8378 (1996).

18. Akasaka, Y., Tsunoda, M., Ogata, T., Ide, T. \& Murakami, K. Direct evidence for leptin-induced lipid oxidation independent of long-form leptin receptor. Biochim. Biophys. Acta. 1801, 1115-1122 (2010).

19. Leung, J.C. et al. The role of leptin and its short-form receptor in inflammation in $\mathrm{db} / \mathrm{db}$ mice infused with peritoneal dialysis fluid. Nephrol. Dial. Transplant. 27, 3119-3129 (2012).

20. Mattioli, B. et al. Leptin as an immunological adjuvant: enhanced migratory and CD8 + Tcell stimulatory capacity of human dendritic cells exposed to leptin. FASEB J. 22, 2012-2022 (2008).

21. Lam, Q.L., Liu, S., Cao, X. \& Lu, L. Involvement of leptin signaling in the survival and maturation of bone marrow-derived dendritic cells. Eur. J. Immunol. 36, 3118-3130 (2006).

22. Mattioli, B., Giordani, L., Quaranta, M.G. \& Viora, M. Leptin exerts an antiapoptotic effect on human dendritic cells via the PI3K-Akt signaling pathway. FEBS Lett. 583, 1102-1106 (2009).

23. Sanchez-Sanchez, N. et al. Chemokine receptor CCR7 induces intracellular signaling that inhibits apoptosis of mature dendritic cells. Blood 104, 619-625 (2004).

24. Yanagawa, Y. \& Onoe, K. CCL19 induces rapid dendritic extension of murine dendritic cells. Blood 100, 1948-1956 (2002).

25. Bell, S.J. et al. Migration and maturation of human colonic dendritic cells. J. Immunol. 166, 4958-4967 (2001).

26. Patterson, S., Gross, J., Bedford, P. \& Knight, S.C. Morphology and phenotype of dendritic cells from peripheral blood and their productive and non-productive infection with human immunodeficiency virus type 1. Immunology 72, 361-367 (1991).

27. Batra, A., Zeitz, M. \& Siegmund, B. Adipokine signaling in inflammatory bowel disease. Inflamm. Bowel Dis. 15, 1897-1905 (2009).

28. Chain, B.M. Current issues in antigen presentation-focus on the dendritic cell. Immunol. Lett. 89, 237-241 (2003).

29. Middel, P., Raddatz, D., Gunawan, B., Haller, F. \& Radzun, H.J. Increased number of mature dendritic cells in Crohn's disease: evidence for a chemokine mediated retention mechanism. Gut 55, 220-227 (2006).

30. Cruickshank, S.M., English, N.R., Felsburg, P.J. \& Carding, S.R. Characterization of colonic dendritic cells in normal and colitic mice. World J. Gastroenterol. 11, 6338-6347 (2005).

31. Xin, H.M., Peng, Y.Z., Yuan, Z.Q. \& Guo, H. In vitro maturation and migration of immature dendritic cells after chemokine receptor 7 transfection. Can. J. Microbiol. 55, 859-866 (2009).

32. Lutz, M.B. \& Schuler, G. Immature, semi-mature and fully mature dendritic cells: which signals induce tolerance or immunity?. Trends Immunol. 23, 445-449 (2002).

33. Seth, S. et al. CCR7 essentially contributes to the homing of plasmacytoid dendritic cells to lymph nodes under steady-state as well as inflammatory conditions. J. Immunol. 186, 3364-3372 (2011).

34. van den, B.M. Dendritic cells break bonds to tolerize. Immunity 27, 544546 (2007).

35. Jung, K.C. et al. In situ induction of dendritic cell-based T cell tolerance in humanized mice and nonhuman primates. J. Exp. Med. 208, 2477-2488 (2011).
36. Stagg, A.J., Hart, A.L., Knight, S.C. \& Kamm, M.A. Microbial-gut interactions in health and disease. Interactions between dendritic cells and bacteria in the regulation of intestinal immunity. Best Pract. Res. Clin. Gastroenterol. 18, 255-270 (2004).

37. Rescigno, M. Dendritic cells in oral tolerance in the gut. Cell Microbiol. 13, 1312-1318 (2011)

38. Rivas-Caicedo, A. et al. Jak3 is involved in dendritic cell maturation and CCR7-dependent migration. PLoS One 4, e7066 (2009).

39. Hopken, U.E. et al. Up-regulation of the chemokine receptor CCR7 in classical but not in lymphocyte-predominant Hodgkin disease correlates with distinct dissemination of neoplastic cells in lymphoid organs. Blood 99, 1109-1116 (2002).

40. Mburu, Y.K., Abe, K., Ferris, L.K., Sarkar, S.N. \& Ferris, R.L. Human beta-defensin 3 promotes NF-kappaB-mediated CCR7 expression and anti-apoptotic signals in squamous cell carcinoma of the head and neck. Carcinogenesis 32, 168-174 (2011).

41. Suzuki, A. et al. CIS3/SOCS3/SSI3 plays a negative regulatory role in STAT3 activation and intestinal inflammation. J. Exp. Med. 193, 471-481 (2001).

42. Musso, A. et al. Signal transducers and activators of transcription 3 signaling pathway: an essential mediator of inflammatory bowel disease and other forms of intestinal inflammation. Inflamm. Bowel Dis. 11, 91-98 (2005).

43. Nishi, Y. et al. Plasma leptin and ghrelin concentrations in patients with Crohn's disease. World J. Gastroenterol. 11, 7314-7317 (2005).

44. Karmiris, K. et al. Circulating levels of leptin, adiponectin, resistin, and ghrelin in inflammatory bowel disease. Inflamm. Bowel Dis. 12, 100-105 (2006).

45. Barbier, M. et al. Overexpression of leptin mRNA in mesenteric adipose tissue in inflammatory bowel diseases. Gastroenterol. Clin. Biol. 27, 987-991 (2003).

46. Makkonen, J. et al. Increased expression of the macrophage markers and of 11 beta-HSD-1 in subcutaneous adipose tissue, but not in cultured monocyte-derived macrophages, is associated with liver fat in human obesity. Int. J. Obes. (Lond.) 31, 1617-1625 (2007).

47. Fawcett, R.L. et al. Tumor necrosis factor-alpha inhibits leptin production in subcutaneous and omental adipocytes from morbidly obese humans. J Clin. Endocrinol. Metab. 85, 530-535 (2000).

48. Panoskaltsis, N., Reid, C.D. \& Knight, S.C. Quantification and cytokine production of circulating lymphoid and myeloid cells in acute myelogenous leukaemia. Leukemia 17, 716-730 (2003).

49. Watson, J.V. Flow Cytometry Data Analysis; Basic Concepts and Statistics. (Cambridge University Press, Cambridge, 2003).

50. Holden, N.J. et al. Dendritic cells from control but not atopic donors respond to contact and respiratory sensitizer treatment in vitro with differential cytokine production and altered stimulatory capacity. Clin. Exp. Allergy 38, 1148-1159 (2008).

51. Patterson, S., Gross, J, Bedford, P. \& Knight, S.C. Morphology and phenotype of dendritic cells from peripheral blood and their productive and non-productive infection with human immunodeficiency virus type 1. Immunology 72, 361-367 (1991).

This work is licensed under the Creative Commons Attribution-NonCommercial-No Derivative Works 3.0 Unported License. To view a copy of this license, visit http:// creativecommons.org/licenses/by-nc-nd/3.0/ 